\title{
Multifluid Eulerian model of an electrospray in a host gas
}

\author{
F. J. Higuera $\uparrow$ \\ E. T. S. Ingenieros Aeronáuticos, UPM, Plaza Cardenal Cisneros 3, 28040 Madrid, Spain
}

An Eulerian multifluid model is used to describe the evolution of an electrospray plume and the flow induced in the surrounding gas by the drag of the electrically charged spray droplets in the space between an injection electrode containing the electrospray source and a collector electrode. The spray is driven by the voltage applied between the two electrodes. Numerical computations and order-of-magnitude estimates for a quiescent gas show that the droplets begin to fly back toward the injection electrode at a certain critical value of the flux of droplets in the spray, which depends very much on the electrical conditions at the injection electrode. As the flux is increased toward its critical value, the electric field induced by the charge of the droplets partially balances the field due to the applied voltage in the vicinity of the injection electrode, leading to a spray that rapidly broadens at a distance from its origin of the order of the stopping distance at which the droplets lose their initial momentum and the effect of their inertia becomes negligible. The axial component of the electric field first changes sign in this region, causing the fly back. The flow induced in the gas significantly changes this picture in the conditions of typical experiments. A gas plume is induced by the drag of the droplets whose entrainment makes the radius of the spray away from the injection electrode smaller than in a quiescent gas, and convects the droplets across the region of negative axial electric field that appears around the origin of the spray when the flux of droplets is increased. This suppresses fly back and allows much higher fluxes to be reached than are possible in a quiescent gas. The limit of large droplet-to-gas mass ratio is discussed. Migration of satellite droplets to the shroud of the spray is reproduced by the Eulerian model, but this process is also affected by the motion of the gas. The gas flow preferentially pushes satellite droplets from the shroud to the core of the spray when the effect of the inertia of the droplets becomes negligible, and thus opposes the well-established electrostatic/inertial mechanism of segregation and may end up concentrating satellite droplets in an intermediate radial region of the spray.

Key words: drops and bubbles, electrohydrodynamic effects

\section{Introduction}

The cone-jet mode of electrostatic atomization allows nearly monodisperse sprays of electrically charged micrometric or nanometric droplets to be generated; see Cloupeau \& Prunet-Foch (1989) and Fernández de la Mora (2007). These sprays are of interest

\footnotetext{
$\dagger$ Email address for correspondence: fhiguera@aero.upm.es
} 
in the pharmaceutical and food industries (Barrero \& Loscertales 2007), and for material processing (Lee et al. 2001), space thrusters (Gamero-Castano 2008) and mass spectrometry (Fenn et al. 1989). The electric charge of the droplets provides the spray with specific properties. It leads to Coulomb repulsion forces that prevent agglomeration and tend to disperse the spray, and may induce an electric field on the spray source that affects its operation. The electric charge of the droplets also allows the spray to be controlled and guided by means of a suitable electric field. The dynamics of sprays of charged droplets has been studied both in vacuum and in a host medium that opposes a resistance to the motion of the droplets, and it is the subject of this paper.

Fernández de la Mora (1992) showed that a monodisperse spray of very small droplets, with negligible inertia, issuing from the tip of a conical equipotential meniscus into a gas at rest takes a conical self-similar form, and computed the angular distributions of spray density and electric current, as well as the angle of the conical meniscus in the presence of the spray. The idealizations used in this analysis are appropriate for an electrospray of a liquid of very high electrical conductivity working at a flow rate near the minimum of the cone-jet mode, for which the droplets are very small and monodisperse.

Gañán-Calvo et al. (1994) carried out the first Lagrangian simulations of a spray of charged droplets moving in quiescent air with important inertial effects. The equations of motion of individual droplets were solved numerically accounting for the effects of the electric field due to the voltage applied between the capillary through which the liquid is injected and a plate collector electrode perpendicular to the capillary, the electrostatic interaction of all of the spray droplets and their images in the collector electrode, and the air drag force. The numerical results were compared with phase Doppler anemometry (PDA) size and velocity measurements for two electrosprays of heptane doped with an antistatic additive. The results showed the size segregation effect first noted by Zeleny (1917), whereby large droplets carrying most of the mass and charge occupy a core region while small droplets shift to the shroud of the spray. Gañan-Calvo et al. (1994) also showed that the average droplet size of polydisperse sprays first decreases and then increases with distance to the needle due to differential accumulation of small and large droplets caused by their different mobilities.

Tang \& Gomez (1994) extensively investigated the structure of a heptane-doped electrospray using PDA to measure droplet sizes, velocities and concentrations, together with flash shadowgraphy to visualize the initial breakup of the liquid ligament into primary and satellite droplets. These authors proposed an electrostatic/inertial explanation of size segregation according to which Coulombic repulsion causes small satellite droplets to rapidly migrate away of the spray axis following any initial perturbation, due to the small mass of these droplets compared with the mass of the primary droplets. Then small droplets are kept at the shroud of the spray when inertial effects become negligible because they follow the radial electric field in this region, which is larger than in the spray core. Tang \& Gomez determined the electric field from the measured droplet size, velocity and concentration, using a momentum balance for the spray. They find that the radial field induced by the charge of the droplets is responsible for the lateral spreading of the spray, while the axial field is due mainly to the voltage applied between the electrodes in the conditions of their experiments. They also measured the gas velocity induced by the entrainment of the spray, which is about one third of the droplet velocity in the droplet generation region and decreases streamwise as the spray broadens. They conclude that the motion of the gas has an important effect on the evolution of the droplets. 
Experimental and numerical techniques similar to those of Tang \& Gomez (1994) and Gañán-Calvo et al. (1994) were used by Hartman et al. (1999) for electrosprays of ethylene glycol. In addition to confirming many of the results commented above, these authors estimated the velocity induced in the gas from the differences between measured droplet velocities and droplet velocities computed with the assumption of a quiescent gas, and found that the effect of the motion of the gas is important in the core of the spray. Hartman et al. also found that the agreement between their numerical and experimental results is best when the electric charge of the droplets is taken to vary as the power 1.5 of their diameter, which coincides with the results of Tang \& Gomez (1994), and that the droplet seeding conditions have an effect on the computed evolution of the spray.

Additional Lagrangian simulations of sprays of charged droplets have been carried out by Wilhelm, Mädler \& Pratsinis (2003), who included the effect of the evaporation of the droplets in the surrounding gas; Oh, Kim \& Kim (2008), who simulated the interaction of the sprays that issue from two sources in tandem; and Deng \& Gomez (2007), who investigated the sprays beyond the extractor electrode of a multiplexed source and the conditions leading to path reversal and fly back of the satellite droplets toward the extractor. These authors also proposed simplified models of the space charge induced field, while Yang et al. (2012) carried out massively parallel computations of multiplexed electrosprays. Grifoll \& Rosell-Llompart (2012) achieved significant reductions of the computer time required by large Lagrangian simulations, without degrading the accuracy of the results, by mean of numerical techniques based on lumping space charge to evaluate Coulombic interactions between distant droplets and using different time steps in regions of different droplet densities.

Eulerian formulations aim at describing the spray as a continuous medium, in terms of macroscopic fields of droplet size, number density and velocity, which are moments of a distribution function. When the inertia of the droplets plays a role, Eulerian formulations require closure models for the macroscopic momentum transfer due to the fluctuations of the droplet velocities about their macroscopic velocity, and also for the momentum exchange with the gas phase, when the motion of the gas is to be taken into account (Landau \& Lifshitz 1981, chapters 3 and 4; Jackson 2000). When they are applicable, Eulerian models give results at a fraction of the cost of Lagrangian simulations. An Eulerian simulation of a monodisperse electrospray was presented by Grace \& Dunn (1996) based on the formulation of Filippov (1991, 1992) for the related problem of the electrostatic deposition of inertial charged particles. Higuera (2012) used an Eulerian model to describe the bidisperse spray beyond the extractor of an electrospray source. This model is extended here to take into account the motion induced in the gas by the drag of the droplets, and it is applied to describe the plume of an electrospray in the common needle-plate electrode configuration.

The paper is organized as follows. An Eulerian model is formulated in $\S 2$ of a bidisperse electrospray made of primary and satellite, electrically charged droplets moving in a gas that is set in motion by the drag of the droplets. The fairly standard numerical method used to solve the gas and droplet equations is briefly described, and a reference case is defined based on the experiments of Gañan-Calvo et al. (1994). The case of a monodisperse spray in a quiescent gas is discussed in $\S 3$. Numerical results for very small fluxes of droplets agree with the view put forward by Tang \& Gomez (1994) of a radial electric field dominated by the charge of the spray and an axial electric field dominated by the voltage applied between the electrodes. The axial field induced by the charged droplets increases with the flux of droplets and overcomes the applied field for a certain critical value of the flux, above which some 
droplets fly back toward the injection electrode and the Eulerian model ceases to be applicable. The value of the critical flux is very much decreased by the image charge of the droplets in the injection electrode, which has not been taken into account in many previous simulations. The results of the Eulerian model are compared with Lagrangian simulations carried out with a standard particle method. The flow of the gas induced by the drag of the droplets is discussed in $\S 4$. It is shown that a gas plume develops at some distance from the injection electrode, whose entrainment causes a flow that suppresses fly back in the conditions of the experiments reviewed above by carrying the droplets across the region of negative axial electric field that appears near the electrode when the flux of droplets is increased. The limit of large droplet-to-gas mass ratio is discussed. Numerical computations of sprays with satellite droplets are presented in $\S 5$. The Eulerian model reproduces the size segregation found in experiments and Lagrangian simulations. It shows that the flow of the gas may have an important effect on size segregation, as it is more effective at carrying satellite droplets than primary droplets towards the axis of the spray, and thus may partially offset the electrostatic/inertial mechanism of Tang \& Gomez (1994) when the inertia of both droplet populations becomes negligible.

\section{Formulation}

Consider a spray of electrically charged, non-evaporating droplets generated by an electrospray working in the cone-jet mode. The liquid to be atomized issues from a metallic capillary tube of inner radius $a$ at a distance $H$ from a collector electrode. Under the action of an electric field due to a voltage $V$ applied between the tube and the collector, the meniscus at the end of the tube takes a conical shape (Taylor 1964) with a thin jet emerging from its tip, as sketched by the contours in the shaded region of figure 1. The jet breaks into electrically charged droplets at some distance from the meniscus, and the spray of droplets is pushed toward the collector by the electric field; see Fernández de la Mora (2007) for a detailed description. The electrospray is assumed to generate $\phi_{p}$ primary droplets of mass $m_{p}$, charge $q_{p}$ and diameter $d_{p}$ per unit time, and $\phi_{s}$ satellite droplets of mass $m_{s}$, charge $q_{s}$ and diameter $d_{s}$ per unit time. The space between the tube and the collector is filled with a gas of density $\rho_{g}$, viscosity $\mu_{g}$ and permittivity $\epsilon_{0}$ which opposes a resistance to the motion of the droplets and is set in motion by their drag.

The electrohydrodynamic processes responsible for the formation of the cone-jet and the breakup of the jet are complex and not fully understood, and they have characteristic scales very different from the scales of the spray. No attempt is made here to simulate these processes together with the spray. Instead, as in previous works, simplified representations of the electrical conditions around the meniscus and of the droplet generation process, based on known properties of the cone-jet, are used to simulate the spray. The elements of these simplified representations are as follows.

(i) Around its tip, the meniscus is known to approach a Taylor cone, which is an exact hydrostatic solution with an equipotential surface. The electric potential induced by a Taylor cone, $\varphi_{T}$ say, whose expression is given after (2.10) below, is imposed as a boundary condition at the fictitious surface of a paraboloid of curvature $4 / a$ at its tip which surrounds the meniscus (dashed contour bounding the shaded region in figure 1, which is thereby excluded from further consideration). For convenience, the outer surface of the metallic capillary tube, which is at the same potential as the meniscus attached to its end, is assumed to be a prolongation of this paraboloid, and the collector electrode is a confocal paraboloid. The needle-plate electrode configuration 


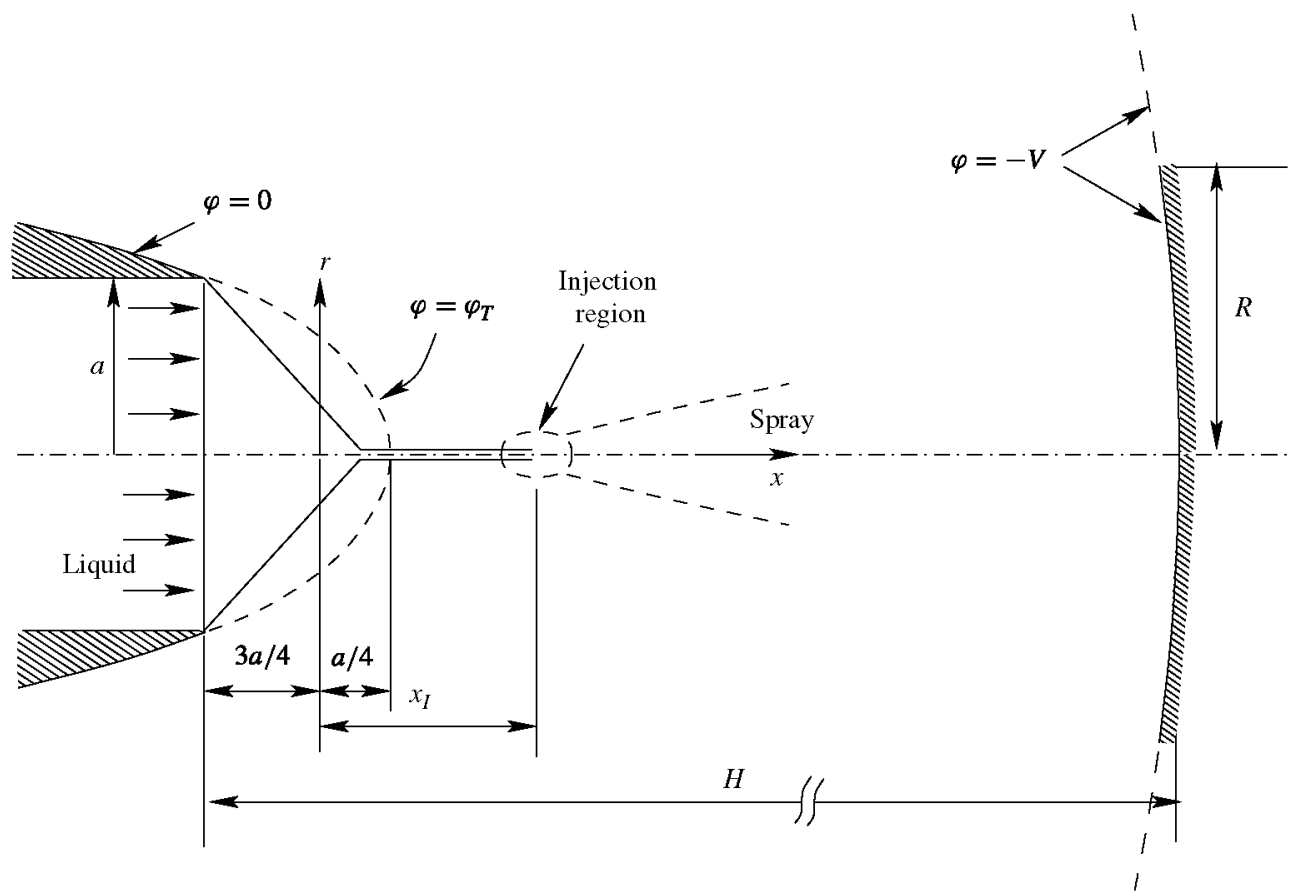

FIGURE 1. Definition sketch.

is thus replaced by a couple of paraboloids, but this is not expected to significantly affect the evolution of the spray when $H \gg a$. Numerical tests show that the solution is also little affected when $\varphi_{T}$ is changed to the constant potential of the capillary. The collector electrode captures the droplets that reach it. This electrode is permeable to the gas except in a central area of radius $R$; see (2.12) below. In what follows, axial distances are measured from the focus of the paraboloids.

(ii) The jet that emerges from the vertex of the meniscus is modelled as a line of charge of strength (charge per unit length) $\left(q_{p} \phi_{p}+q_{s} \phi_{s}\right) / v_{I}$ projecting from the tip of the paraboloid that encloses the meniscus; see (2.3) below. Here $v_{I}$ is the initial velocity of the spray droplets generated by the breakup of the jet. In the continuous model of the spray discussed below, the generation of these droplets is modelled by means of source terms in the mass and momentum conservation equations (2.1) below, which are arbitrarily assumed to have Gaussian distributions with standard deviation of the order of the diameter of the primary droplets, $d_{p}$, centred at the end of the line of charge modelling the jet (at $x=x_{I}$ ).

A simple Eulerian multifluid model (Jackson 2000) is used to describe the dynamics of the spray and the gas. The treatment of the spray as a continuous dilute phase relies on the conditions that the mean distance between droplets is small compared with any characteristic macroscopic length, and large compared with the diameter of the droplets. The first condition allows to define macroscopic number densities and velocities for the primary and satellite droplets, $\left(n_{p}, \boldsymbol{v}_{p}\right)$ and $\left(n_{s}, \boldsymbol{v}_{s}\right)$, respectively, which are continuous, non-fluctuating magnitudes to be computed together with the gas velocity and pressure, $\boldsymbol{v}_{g}$ and $p_{g}$. The second condition allows to neglect mechanical contacts between charged droplets, which interact only through the electric and flow fields they induce. 
Droplet velocity fluctuations about the macroscopic velocities $v_{i}$, with $i=p, s$ for primary and satellite droplets, lead to stress tensors whose divergences should be included in the macroscopic momentum equations for the disperse (droplet) phases (Jackson 2000). As in previous works (Filippov 1991, 1992; Tang \& Gomez 1994; Grace \& Dunn 1996), these stresses will be left out for the following reasons. Injection and hydrodynamic interactions are not expected to generate large velocity fluctuations, even though the inertia of the droplets matters in an initial stage of their evolution (the Stokes numbers $m_{i} v_{I} / d_{i} c_{f_{i}}$, where $c_{f_{i}}$ are the friction coefficients of the droplets, are fairly large), because all of the droplets are taken to start with the same velocity $v_{I}$, pointing along the symmetry axis, and the motion of the gas is due only to the drag of the droplets. Electric forces lead to large velocity fluctuations in conditions when the electric field induced by the charged droplets causes a fraction of these droplets to fly back toward the spray source. While the simple Eulerian model of this section is not applicable in such conditions, previous results (Higuera 2012) show that the electric-field-induced velocity fluctuations are not important in the absence of fly back, and the Eulerian model can even predict the onset of fly back.

The gas is treated as incompressible. Since the droplet-to-gas volume ratio is very small, the droplets affect the gas only through the drag force due to their relative motion. Assuming, in addition, that the Reynolds number of this relative motion and the gas-to-liquid density ratio are small, the stationary mass and momentum conservation equations for the particle populations and the gas are (Jackson 1997, 2000; Zhang \& Prosperetti 1997)

$$
\begin{gathered}
\boldsymbol{\nabla} \cdot\left(n_{i} \boldsymbol{v}_{i}\right)=S_{i}, \quad m_{i} \boldsymbol{\nabla} \cdot\left(n_{i} \boldsymbol{v}_{i} \boldsymbol{v}_{i}\right)=n_{i} q_{i} \boldsymbol{E}-n_{i} c_{f_{i}}\left(\boldsymbol{v}_{i}-\boldsymbol{v}_{g}\right)+m_{i} S_{i} \boldsymbol{v}_{I}, \\
\nabla \cdot \boldsymbol{v}_{g}=0, \quad \rho_{g} \boldsymbol{v}_{g} \cdot \nabla \boldsymbol{v}_{g}=-\nabla p_{g}+\mu_{g} \nabla^{2} \boldsymbol{v}_{g}+n_{p} c_{f_{p}}\left(\boldsymbol{v}_{p}-\boldsymbol{v}_{g}\right)+n_{s} c_{f_{s}}\left(\boldsymbol{v}_{s}-\boldsymbol{v}_{g}\right),
\end{gathered}
$$

where $c_{f_{i}}=3 \pi \mu_{g} d_{i}$ for Stokes drag (corrections for non-small Reynolds numbers of the droplet-to-gas flow are available; see Clift, Grace \& Weber (1978)), and $S_{i}$ are the source terms mentioned above. The macroscopic electric field that appears in the momentum equations for the droplets is $E=-\nabla \varphi$ with

$$
\nabla^{2} \varphi=-\frac{q_{p} n_{p}+q_{s} n_{s}}{\epsilon_{0}}-\frac{q_{j e t}}{\epsilon_{0}},
$$

where $q_{j e t}=2 \delta\left(r^{2}\right)\left(q_{p} \phi_{p}+q_{s} \phi_{s}\right) / \pi v_{I}$ is the charge distribution mimicking the jet on a segment of the symmetry axis, with $\delta$ the Dirac delta function.

Dimensionless variables can be introduced by scaling distances with the inner radius of the capillary tube, $a$, the electric potential with the voltage $V$ applied between capillary and collector, velocities with $v_{0}=\left(q_{p} V / m_{p}\right)^{1 / 2}$, pressure variations with $\rho_{g} v_{0}^{2}$, and number densities with $n_{0}=\epsilon_{0} V / q_{p} a^{2}$, which is the number density for which the space-charge-induced field would be of the order of the field due to the applied voltage in a region of characteristic size $a$ around the end of the capillary tube. Denoting dimensionless variables with the same symbols used before for their dimensional counterparts, the governing equations take the form

$$
\begin{gathered}
\nabla \cdot\left(n_{p} \boldsymbol{v}_{p}\right)=\Lambda S_{p}, \quad \boldsymbol{v}_{p} \cdot \nabla \boldsymbol{v}_{p}=\boldsymbol{E}-\Pi\left(\boldsymbol{v}_{p}-\boldsymbol{v}_{g}\right)+\Lambda S_{p} \frac{\boldsymbol{v}_{I}-\boldsymbol{v}_{p}}{n_{p}}, \\
\nabla \cdot\left(n_{s} \boldsymbol{v}_{s}\right)=\phi_{r} \Lambda S_{p}, \quad \boldsymbol{v}_{s} \cdot \nabla \boldsymbol{v}_{s}=\frac{q_{r}}{m_{r}} \boldsymbol{E}-\frac{c_{r}}{m_{r}} \Pi\left(\boldsymbol{v}_{s}-\boldsymbol{v}_{g}\right)+\phi_{r} \Lambda S_{p} \frac{\boldsymbol{v}_{I}-\boldsymbol{v}_{s}}{n_{s}}, \\
\nabla \cdot \boldsymbol{v}_{g}=0, \quad \boldsymbol{v}_{g} \cdot \nabla \boldsymbol{v}_{g}=-\nabla p_{g}+\frac{1}{R e} \nabla^{2} \boldsymbol{v}_{g}+F \Pi\left\{n_{p}\left(\boldsymbol{v}_{p}-\boldsymbol{v}_{g}\right)+c_{r} n_{s}\left(\boldsymbol{v}_{s}-\boldsymbol{v}_{g}\right)\right\},
\end{gathered}
$$




$$
\boldsymbol{E}=-\nabla \varphi, \quad \nabla^{2} \varphi=-n_{p}-q_{r} n_{s}-q_{j e t},
$$

where the momentum equations in (2.4) and (2.5) are non-conservative forms of those in (2.1). The source term $S_{p}$ is taken to be

$$
S_{p}=\frac{1}{\pi^{3 / 2} \sigma^{3}} \exp \left[-\frac{\left(x-x_{1}\right)^{2}}{\sigma^{2}}-\frac{r^{2}}{\sigma^{2}}\right],
$$

which satisfies the normalization condition $\int S_{p} 2 \pi r \mathrm{~d} x \mathrm{~d} x=1, S_{s}=\phi_{r} S_{p}$ and $q_{j e t}=$ $2 \delta\left(r^{2}\right)\left(1+q_{r} \phi_{r}\right) \Lambda / \pi v_{I}$. Hereafter $x$ and $r$ are cylindrical coordinates along and normal to the symmetry axis. The 13 dimensionless parameters that appear in these equations and the boundary conditions below are

$$
\left.\begin{array}{l}
\Lambda=\frac{m_{p}^{1 / 2} q_{p}^{1 / 2} \phi_{p}}{\epsilon_{0} V^{3 / 2}}, \quad \Pi=\frac{c_{f_{p}} a}{m_{p}^{1 / 2} q_{p}^{1 / 2} V^{1 / 2}}, \quad R e=\frac{\rho_{g} q_{p}^{1 / 2} V^{1 / 2} a}{\mu_{g} m_{p}^{1 / 2}}, \quad F=\frac{\epsilon_{0} m_{p} V}{\rho_{g} q_{p} a^{2}}, \\
v_{I}, \quad x_{I}, \quad H, \quad \sigma, \quad R, \quad m_{r}=\frac{m_{s}}{m_{p}}, \quad q_{r}=\frac{q_{s}}{q_{p}}, \quad c_{r}=\frac{c_{f_{s}}}{c_{f_{p}}}, \quad \phi_{r}=\frac{\phi_{s}}{\phi_{p}} .
\end{array}\right\}
$$

Here $\Lambda$ is the flux of primary droplets $\left(\phi_{p}\right)$ scaled with $n_{0} v_{0} a^{2}, \Pi$ is the friction coefficient of the primary droplets scaled with $q_{p} V / a v_{0}, R e=\rho_{g} v_{0} a / \mu_{g}$ is a Reynolds number based on $v_{0}$ and $a$, and $F=m_{p} n_{0} / \rho_{g}$ is a measure of the droplets-to-gas mass ratio. Parameter $v_{I}$ is the initial velocity of the droplets scaled with $v_{0}$, and $x_{I}, H, \sigma, R$ are the dimensionless counterparts of parameters introduced before, all of them scaled with $a$. Finally $m_{r}, q_{r}, c_{r}$ and $\phi_{r}$ are satellite-to-primary ratios of droplet mass, electric charge, friction coefficient and flux.

Equations (2.4)-(2.7) are to be solved with the boundary conditions

$$
\boldsymbol{v}_{p}=\boldsymbol{v}_{s}=\boldsymbol{v}_{g}=0, \quad \varphi=\left\{\begin{array}{rr}
0, & r>1 \\
\varphi_{T}, & r<1
\end{array} \text { at } x=\frac{1}{4}-r^{2},\right.
$$

where $x=1 / 4-r^{2}$ is the paraboloidal surface of the tube and its fictitious extension, with $x=-3 / 4$ at the end of the tube and $x=1 / 4$ at the tip of the paraboloid, and

$$
\varphi_{T}=b\left[\left(x-x_{v}\right)^{2}+r^{2}\right]^{1 / 2} P_{1 / 2}\left\{\left(x-x_{v}\right) /\left[\left(x-x_{v}\right)^{2}+r^{2}\right]^{1 / 2}\right\},
$$

with $x_{v}=0.1104 \ldots, b$ a constant and $P_{1 / 2}(\cdot)$ a Legendre function of degree $1 / 2$, is the potential outside a Taylor cone with apex at $x_{v}$ (Taylor 1964);

$$
\begin{gathered}
\boldsymbol{v}_{g}=0, \quad \varphi=-1 \quad \text { at } x=\xi_{0}^{2} / 4-r^{2} / \xi_{0}^{2}, r<R, \\
\boldsymbol{v}_{g} \times \boldsymbol{n}_{c}=0, \quad \boldsymbol{n}_{c} \cdot \nabla \boldsymbol{\omega}_{g}=0, \quad \varphi=-1 \quad \text { at } x=\xi_{0}^{2} / 4-r^{2} / \xi_{0}^{2}, r>R,
\end{gathered}
$$

where $x=\xi_{0}^{2} / 4-r^{2} / \xi_{0}^{2}$, with $\xi_{0}^{2}=4 H-3$, is the surface of the collector electrode, which is impermeable to the gas in the central part $(r<R), \boldsymbol{n}_{c}$ is a vector normal to the collector, $\omega_{g}=\nabla \times \boldsymbol{v}_{g}$ is the vorticity of the gas and the first two conditions $(2.12 b)$ are intended to approximately mimic a grid permeable to the gas for $r>R$; and

$$
\boldsymbol{v}_{g} \times \boldsymbol{n}_{l}=\boldsymbol{\omega}_{g}=0, \quad \boldsymbol{n}_{l} \cdot \nabla \varphi=0 \quad \text { at } x=r^{2} / \eta_{0}^{2}-\eta_{0}^{2} / 4,
$$

where $x=r^{2} / \eta_{0}^{2}-\eta_{0}^{2} / 4$, with $\eta_{0} \gg 1$ constant, is a far fictitious surface bounding laterally the computational domain, and $\boldsymbol{n}_{l}$ is a vector normal to this surface. The value $\eta_{0}=\xi_{0}-1$ is used in the numerical computations. Numerical tests show that the precise form of the boundary conditions (2.13) has no important effect on the solution. 


$\begin{array}{llllllll} & \Lambda & \Pi & R e & F & v_{I} & x_{I} & H \\ \text { G-C } & 0.0819 & 1.3804 & 3.971 & 5.583 & 0.634 & 0.870 & 60 \\ \text { T-G } & 0.1082 & 0.8104 & 1.884 & 22.937 & 0.958 & 9.428 & 133.3\end{array}$

TABLE 1. Dimensionless parameters for the experiments of Gañán-Calvo et al. (1994) (G-C), which are taken as reference values in this paper, and Tang \& Gomez (1994) (T-G).

For the numerical treatment, the non-stationary forms of the conservation equations are marched in time until the solution converges to a stationary state. The nonstationary forms are obtained adding the terms $\partial n_{p} / \partial t$ and $\partial \boldsymbol{v}_{p} / \partial t$ to the left-hand sides of (2.4), $\partial n_{s} / \partial t$ and $\partial \boldsymbol{v}_{s} / \partial t$ to the left-hand sides of (2.5), and $\partial \boldsymbol{v}_{g} / \partial t$ to the left-hand side of the momentum equation in (2.6). Axisymmetric solutions are sought, for which the stream function-vorticity formulation for the gas equivalent to (2.6) is used. The equations are written in paraboloidal coordinates $(\xi, \eta)$, with $x=\left(\xi^{2}-\eta^{2}\right) / 4$, $r=\xi \eta / 2$, so that the surfaces of the tube and the collector electrode become $\xi=1$ and $\xi=\xi_{0}$, respectively; they are discretized using second-order finite differences, upwind for the convection terms and centred for all other terms; and are integrated in time with a fourth-order Runge-Kutta method.

No attempt is made to explore the 13-dimensional parameter space of the system. Instead, a reference case is defined on the basis of the experiments of Gañan-Calvo et al. (1994) and Tang \& Gomez (1994), and a limited exploration is made changing some parameters from their reference values, one at a time. The values of the first seven parameters (2.9) are given in table 1 for the two experiments cited. As can be seen, these parameters are not very different in the two experiments. The main difference is in $x_{I}$, the distance from the origin to the average position of the jet breakup scaled with the inner radius of the tube, which is very large in the experiment of Tang \& Gomez, reflecting a long jet. The value of $F$ is also larger in this experiment than in the experiment of Gañan-Calvo et al., due mainly to the smaller radius of the tube $(0.225 \mathrm{~mm}$ versus $0.5 \mathrm{~mm})$. In what follows, the values of the parameters in the experiment of Gañan-Calvo et al. (1994) are used to define the reference case. The standard deviation $\sigma$ in the source term (2.8) is taken equal to the diameter of the droplets reported for this experiment, which gives $\sigma=0.076$ and $R=15$.

The last four parameters in (2.9) are satellite-to-primary droplet property ratios. On the basis of the results of Tang \& Gomez (1994), the ratio of droplet diameters is taken to be $1 / 3$, and the ratio of electric charges is taken to scale with the power $3 / 2$ of the diameter ratio. This gives $m_{r}=(1 / 3)^{3}, q_{r}=(1 / 3)^{3 / 2}$ and $c_{r}=1 / 3$. Finally, $\phi_{r}=1$ when satellite droplets are present.

The Eulerian model of this section can be extended in principle to deal with other problems, including multiplexed electrospray sources. Simulation of the interaction of the sprays behind the extractor of a multiplexed source may require additional pressure forces in the momentum equations for the droplets, and will lead to a three-dimensional numerical problem that is considerably more complex than the axisymmetric configurations discussed in the remainder of this paper, although the important issue of droplet fly back could perhaps be decided by a local analysis of the vicinity of the extractor orifices. 


\section{Monodisperse spray in a gas at rest}

This and the following sections deal with monodisperse sprays, without satellite droplets. These correspond to setting $\phi_{r}=0$, which leads to $n_{s}=0$, so that (2.5) drops out of the problem and the solution depends only on the first nine parameters in (2.9). In this section we consider the much studied case of a spray in a gas at rest. The velocity of the gas induced by the spray is expected to be small compared with the velocity of the droplets when $F$ is small (or when $\Lambda F$ is small in cases with very small droplet fluxes, for which the right-hand side of the Poisson equation in (2.7) can be neglected everywhere in the interelectrode space). The problem reduces then to (2.4) and (2.7), with $\boldsymbol{v}_{g}=0$, plus the relevant boundary conditions (2.10)-(2.13). The solution depends only on the six dimensionless parameters $\Lambda, \Pi, v_{I}, x_{I}, H$ and $\sigma$. Even though this problem is simpler than the general problem of the previous section, and solutions can be compared with the results of Lagrangian simulations in the literature, the relatively large value of $F$ in the reference case suggests that the motion of the gas plays an important role in the evolution of the spray in the experiments of Gañán-Calvo et al. (1994) and Tang \& Gomez (1994), and in many others. Analysis of the motion of the gas is postponed to the following section.

Figure 2 shows some contours of constant droplet density (solid) and streamlines of the flow of droplets (dashed) for various values of $\Lambda$ with other parameters set to their reference values in the first row of table 1 . The value of $v_{p_{x}}-E_{x} / \Pi$ is above $20 \%$ of its maximum in the hatched region, which is taken to be the region where the effect of the inertia of the droplets (left-hand side of the momentum equation in (2.4)) is important. The electric field induced by the charged droplets is everywhere small compared with the electric field due to the applied voltage for the smallest value of $\Lambda$ for which results are displayed; $\Lambda=0.001$ in figure $2(a)$. Then the droplets follow the applied field when their inertia ceases to matter. However, since $v_{I}=O(1)$ and the velocity $\boldsymbol{E} / \Pi$ induced by the applied field is also of order unity around the injection electrode, no abrupt transition occurs when the inertia of the droplets ceases to matter. Far from the injection electrode, the electric field is that of a paraboloidal needle, which gives $E_{x}=1 /\left(x \ln \xi_{0}^{2}\right)$ around the symmetry axis (see, e.g., Landau \& Lifshitz 1993, chapters 1 and 2), and the droplets are confined within a streak whose characteristic thickness, $\delta_{p}$, can be estimated as follows. Mass conservation requires $n_{p} v_{p_{x}} \delta_{p}^{2} \sim \Lambda$ in the streak, where $v_{p_{x}}=E_{x} / \Pi$. The electric charge per unit length of the streak is $n_{p} \delta_{p}^{2} \sim \Lambda / v_{p_{x}}$, which induces a dimensionless radial field of order $n_{p} \delta_{p}$ and a radial velocity $v_{p_{r}} \sim n_{p} \delta_{p} / \Pi \sim \Lambda /\left(E_{x} \delta_{p}\right)$ at the streak. Finally, the kinematic condition $\delta_{p} / v_{p_{r}} \sim x / v_{p_{x}}$ gives $\delta_{p} \sim\left(\Lambda \Pi x^{3}\right)^{1 / 2}$, where the logarithmic factor $\ln \xi_{0}^{2}$ has been omitted. The $3 / 2$ power law for the radius of the streak is approximately realized in part of the interelectrode space in figure 2(a), although the field of the image charge in the collector checks the growth of the streak when this electrode is approached; see the inset of this figure. A spray spreading under the action of the radial field induced by its own charge in an axial field dominated by the applied voltage agrees with the results of Tang \& Gomez (1994) and others.

The axial field induced by the charged droplets increases with $\Lambda$. The results in figure $2(b)$, for $\Lambda=0.008$, show that the spray opens significantly toward the end of the hatched region, where the effect of the inertia of the droplets begins to decline. When $\Lambda$ increases, the electric field at the symmetry axis (solid curves in figure 3 ) develops a local minimum and a maximum slightly downstream of the injection region centred at $x_{I}$. The minimum electric field becomes negative for $\Lambda \approx 0.098$. For $\Lambda=0.0107$ (figure $2 c$ ), the electric field points toward the injection electrode in the 

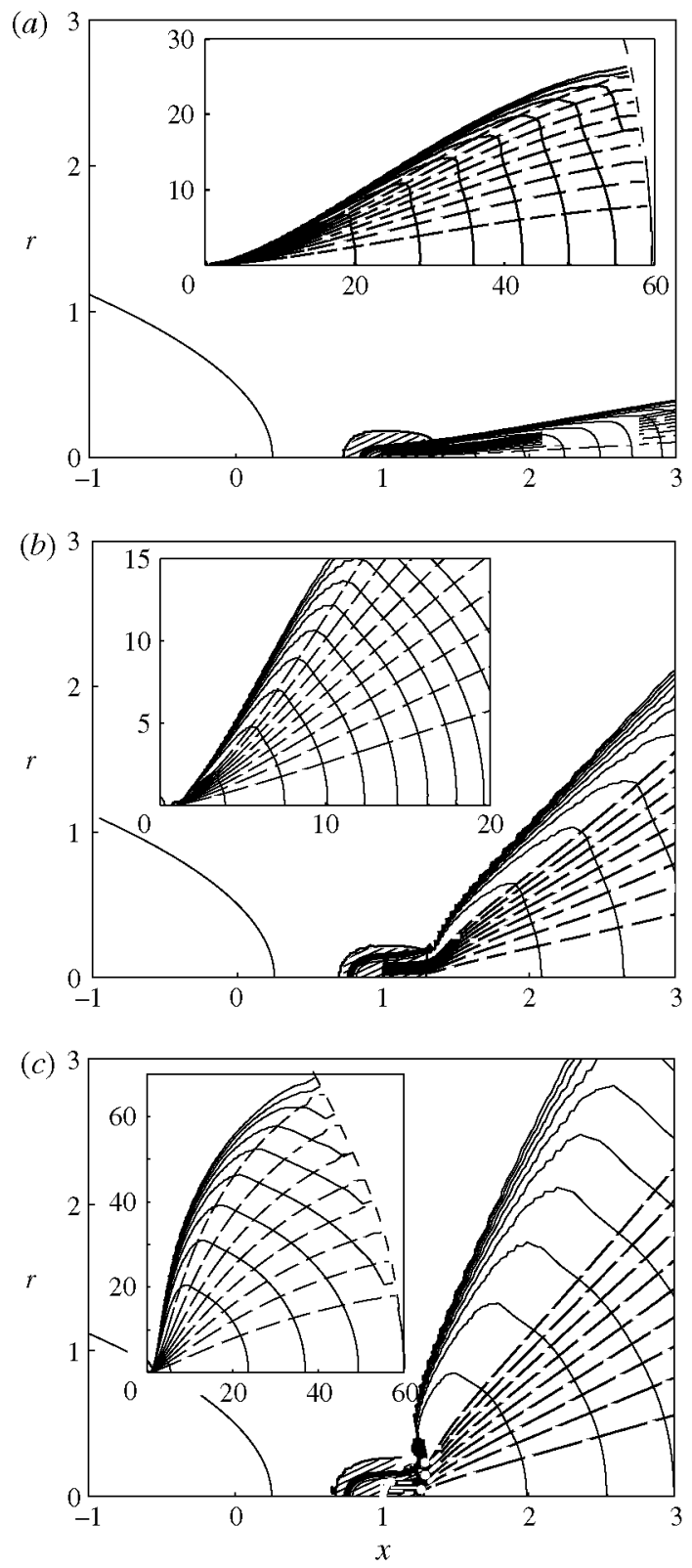

FIGURE 2. (Colour online) Contours of $n_{p}$ (solid, black) and streamlines of the flow of droplets (dashed, shown in red online) in the vicinity of the injection electrode for $(a)$ $\Lambda=0.001$, $(b) \Lambda=0.008$ and $(c) \Lambda=0.0107$. Other parameters have their reference values. The insets show the evolution of the spray farther from the electrode, with the paraboloidal collector electrode indicated by dashed lines at the right-hand sides of the insets in $(a)$ and $(c)$. Shown are 10 equispaced contours of $n_{p}^{-1}$ between 3.33 and 33.33 in $(a)$ and between 10 and 100 in $(b)$ and $(c)$. Hatched: region where $v_{p_{x}}-E_{x} / \Pi$ is above $20 \%$ of its maximum. In $(c)$, the small dotted contour (shown in green online) is the boundary of the region where $E_{x}<0$, and $v_{p x}<0$ in the tiny shaded region (shown in red online). 


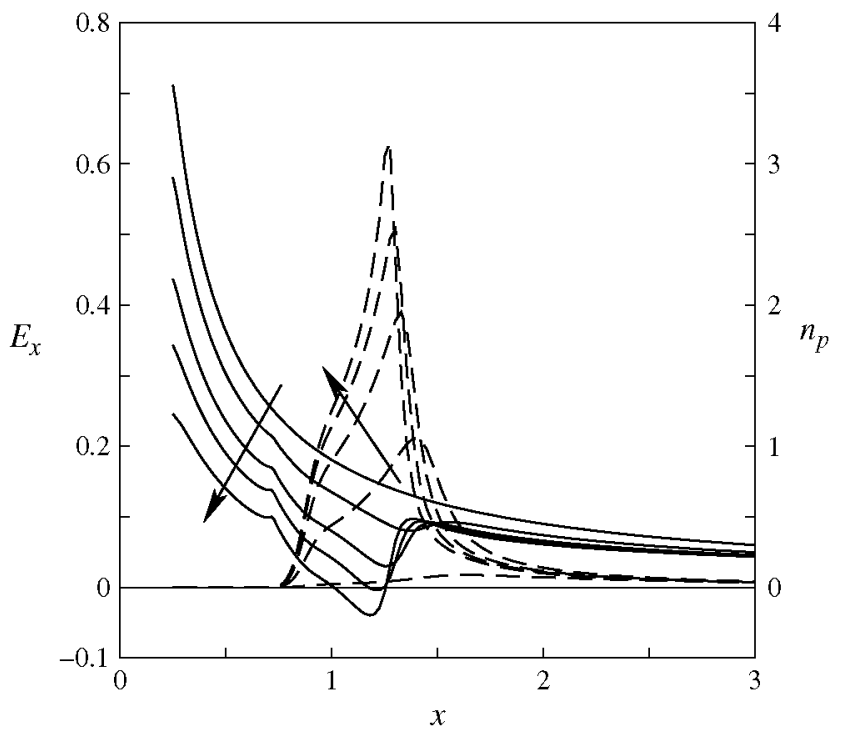

FIGURE 3. (Colour online) Electric field ( $E_{x}$, solid) and droplet number density $\left(n_{p}\right.$, dashed, right-hand side scale) along the symmetry axis for $\Lambda=0.0002,0.004,0.008,0.0100$ and 0.0108 , increasing as indicated by the arrows. Other parameters have their reference values.

small region bounded by the dotted contour, and negative droplet velocities (toward the electrode) appear in the smaller shaded region (shown in red online). This marks the beginning of fly back and the breakdown of the Eulerian model introduced in the previous section. A numerical solution fails to exist when $\Lambda$ is slightly increased.

To account for these results, consider first the electric field induced by the charged droplets in the interelectrode space, $\boldsymbol{E}_{s c}=-\nabla \varphi_{s c}$ with $\nabla^{2} \varphi_{s c}=-n_{p}$ and $\varphi_{s c}=0$ at both electrodes. As could have been expected, $\boldsymbol{E}_{s c}$ evaluated from the solution of this problem points toward the nearest electrode in the vicinity of either the collector or the injection electrode, and changes sign somewhere in between. Furthermore, since the injection electrode appears as a needle when seen from a distance $x$ such that $1 \ll x \ll H$, the field $E_{s c}=\left|\boldsymbol{E}_{s c}\right|$ is locally of the form $E_{s c} \sim C / x$ with $C$ constant (see, e.g., Landau \& Lifshitz 1993, chapters 1 and 2).

The droplets nearly follow the electric field $\left(\boldsymbol{v}_{p}=\boldsymbol{E} / \Pi\right)$ in most of the interelectrode space. When the space-charge-induced field is of the order of the total field, this velocity is $v_{p} \sim E_{s c} / \Pi$ and the order of magnitude of the number density of droplets at a distance $x=O(H)$ from the injection electrode can be estimated from the droplet flux conservation condition as $n_{p} v_{p} H^{2} \sim \Lambda$. Carrying this estimate to the Poisson equation of the previous paragraph $\left(E_{s c} / H \sim n_{p}\right)$ gives $E_{s c} \sim(\Lambda \Pi / H)^{1 / 2}$, and matching with the expression of $E_{s c}$ near the injection electrode requires that $C / x \sim(\Lambda \Pi / H)^{1 / 2}$ for $x=O(H)$, whence $C \sim(\Lambda \Pi H)^{1 / 2}$. The electric field due to the voltage applied between the electrodes is $E_{h} \sim 1 / x$ up to a logarithmic factor. Therefore, $E_{s c}$ and $E_{h}$ balance each other near the injection electrode when $C \sim 1$, which amounts to $\Lambda \sim \Lambda_{c}=1 /(\Pi H)$. Larger values of $\Lambda$ would lead to an electric field that points toward the injection electrode and causes the charged droplets to fly back toward this electrode. The value of $\Lambda_{c}$ is 0.012 for the reference values of $\Pi$ and $H$, which is not far from the value of $\Lambda$ for which fly back first occurs 
and above which numerical solutions could not be computed. Additional computations to be discussed below were carried out with $\Pi=1.5440$ and $H=20.5$, for which $\Lambda_{c}=0.0332$, which again is not far from the maximum $\Lambda \approx 0.04$ for which a numerical solution could be computed in this case.

Values of $\Lambda$ of order $\Lambda_{c}$ make $E_{S c}$ of the order of $E_{h}$ in the bulk of the interelectrode space, for $x=O(H)$, an estimation confirmed by the numerical results. A self-similar conical spray should be expected in regions where $E_{s c}$ points away from the injection electrode and is large compared with $E_{h}$ (Fernández de la Mora 1992). Even though the latter condition is never realized for the problem at hand, the spray is nearly conical in a region of the interelectrode space; see the inset of figure $2(b)$.

Consider now the initial region of the spray. Since the axial electric field in this region is small when $\Lambda$ becomes of the order of $\Lambda_{c}$, the air resistance is the dominant axial force acting on the droplets. This force decelerates the droplets, increasing the residence time during which the radial field induced by the droplets pushes them away from the symmetry axis and increases the radius of the spray. In the absence of an axial electric field, the momentum equation for the droplets in (2.4) projected in the nearly axial direction of their velocity reduces to $v_{p_{x}} \partial v_{p_{x}} / \partial x=-\Pi v_{p_{x}}$, whose solution is $v_{p_{x}}=v_{I}-\Pi\left(x-x_{i}\right)$ for a droplet starting at $x_{i}$ with a velocity $v_{I}$. This droplet would stop at a distance $x_{S}=x_{i}+v_{I} / \Pi$. In the presence of a weak axial field, $E_{x} \ll 1$, the velocity $v_{p_{x}}$ still decreases linearly with $x$ down to distances of order $E_{x} / \Pi$ from $x_{S}$, where $\Pi v_{p_{x}}=O\left(E_{x}\right)$. On the other hand, evaluating the radial electric field induced by the droplets at the lateral boundary of the spray, $r=r_{c}(x)$ say, as $E_{r}=\Lambda /\left(2 \pi v_{p_{x}} r_{c}\right)$, the radial component of the momentum equation in (2.4) at this boundary, and the kinematic condition for $r_{c}$, read

$$
v_{p_{x}} \frac{\partial v_{p_{x}}}{\partial x}=-\Pi v_{p_{r}}+\frac{\Lambda}{2 \pi v_{p_{x}} r_{c}} \quad \text { and } \quad \frac{\mathrm{d} r_{c}}{\mathrm{~d} x}=\frac{v_{p_{r}}}{v_{p_{x}}},
$$

whose solution for small values of $v_{p_{x}}$ is of the form $v_{p_{r}} \approx\left(\Lambda / 6 \pi v_{p_{x}}\right)^{1 / 2}$ and $r_{c} \approx\left(2 \Lambda / 3 \pi v_{p_{x}}\right)^{1 / 2} / \Pi$. The divergence of $r_{c}$ at the stopping point accounts for the opening of the spray in figures $2(b)$ and $2(c)$ following a region of quasiunidirectional droplet motion.

\subsection{Effect of the injection electrode}

The maximum value of $\Lambda$ for which a solution without fly back could be computed is much smaller than the value of $\Lambda$ in the experiments of Tang \& Gomez (1994). It is also smaller than the values of $\Lambda$ for which numerical solutions have been computed by Gañán-Calvo et al. (1994), Hartman et al. (1999), Wilhelm et al. (2003) and Grifoll \& Rosell-Llompart (2012), among others, using Lagrangian simulations of the spray. Differing in other important respects, all of these numerical works compute the Coulomb forces between pairs of droplets without accounting for the image charges that the droplets induce in the injection electrode. The presence of this electrode, and of the meniscus attached to its end, is of course accounted for in the computation of the harmonic electric field due to the applied voltage, but the injection electrode ceases to be an equipotential when the field of the charged droplets but not their images are added to the harmonic field. Image charges in the collector electrode are included in the computations of Gañán-Calvo et al. (1994), Wilhelm et al. (2003) and Grifoll \& Rosell-Llompart (2012).

To gain some insight into the magnitude of the field due to the image charges, the electric potential of a point charge $Q$ in the interelectrode space has been computed 
by solving (2.7) with the right-hand side replaced by $-Q \delta\left(\boldsymbol{x}-\boldsymbol{x}_{0}\right)$ and $\varphi=0$ at both electrodes. Here $\delta$ is the Dirac delta function and $\boldsymbol{x}_{0}$ is a point of the symmetry axis. When $\boldsymbol{x}_{0}$ approaches the tip of the injection electrode, the field induced at $\boldsymbol{x}_{0}$ by the image charge diverges as $-Q /\left[8 \pi\left(\left|\boldsymbol{x}_{0}\right|-1 / 4\right)^{2}\right]$, which is the result for a point charge in front of a planar electrode (the minus sign meaning that the field points toward the injection electrode). The strength of the induced field rapidly decreases when $\left|\boldsymbol{x}_{0}\right|$ increases, is zero for $\left|\boldsymbol{x}_{0}\right| \approx 22$ and becomes positive (toward the collector) when the point charge is farther from the injection electrode. For $\left|\boldsymbol{x}_{0}\right|=2 x_{I}=1.74$, the induced field is $-6.36 \times 10^{-3} Q=-0.127$, taking $Q=20$, which is the order of the dimensionless charge within the dotted contour in figure $2(c)$. This field is of the order of the harmonic field at the same point, and also of the order of the total field in figure 3 .

These results are for a paraboloidal injection electrode. They are approximations to the field of the real image charge, because the leading part of this electrode is a representation of the electrospray source rather than the meniscus itself; see figure 1 . Approximate computations for a point charge in front of a capillary with a conical tip, which models an electrically stretched meniscus attached to the capillary, show that the paraboloidal electrode overpredicts the field of the image charge for small values of $\left|\boldsymbol{x}_{0}\right|$. For example, these computations give an induced field $-3.90 \times 10^{-3} Q$ for $\left|x_{0}\right|=2 x_{I}$, to be compared to $-6.36 \times 10^{-3} Q$ above. The difference may partially account for the small maximum value of $\Lambda$ mentioned at the beginning of this section, but it is clear that the image charge in the injection electrode has an important effect on the electric field.

Here, to approach the conditions of the computations that neglect this image charge and to assess its effect, additional computations have been carried out in which the electric potential is written as $\varphi=\varphi_{h}+\varphi_{s c}$ and computed in two steps. First, the harmonic potential $\varphi_{h}$ is computed solving (2.7) with $n_{p}$ and $n_{s}$ on the righthand side set to zero and the relevant boundary conditions $(2.10)-(2.13)$. Then the electric potential of the space charge is computed solving $\nabla^{2} \varphi_{s c}=-n_{p}$ with the injection electrode removed, i.e. in a domain bounded only by the collector electrode $x=\xi_{0}^{2} / 4-r^{2} / \xi_{0}^{2}$ and the lateral boundary $x=r^{2} / \eta_{0}^{2}-\eta_{0}^{2} / 4$, and with $\varphi_{s c}=0$ in (2.12). The results of these computations show that fly back is postponed to fairly large values of $\Lambda$ when the image charge in the injection electrode is omitted, as could be expected since this charge attracts the droplets toward the electrode.

As a further test of the Eulerian model, the results are compared with a Lagrangian simulation carried out with a standard axisymmetric particle method (Birdsall \& Langdon 1981; Hockney \& Eastwood 1988) in which the trajectories and velocities, $\boldsymbol{x}_{j}(t)$ and $\boldsymbol{v}_{j}(t)$, of identical particles of mass $m_{p}$ and charge $q_{p}$, which are injected with initial velocity $\boldsymbol{v}_{I}$ at points sampled from the Gaussian distribution (2.8), are computed by solving the equations of motion $\mathrm{d} \boldsymbol{x}_{j} / \mathrm{d} t=\boldsymbol{v}_{j}$ and $m_{p} \mathrm{~d} \boldsymbol{v}_{j} / \mathrm{d} t=-c_{f_{p}} \boldsymbol{v}_{j}+q_{p} \boldsymbol{E}\left(\boldsymbol{x}_{j}\right)$ with $\boldsymbol{E}=-\nabla \varphi$ and $\varphi$ computed as in the previous paragraph. The charge density on the right-hand side of $(2.3)$ is $q_{p} n_{p} / \epsilon_{0}$, with $n_{p}$ evaluated by linear, particle-in-cell weighting of the cloud of droplets on the grid used to discretize the Poisson equation (see Birdsall \& Langdon 1981). The evolution of each particle is followed until it reaches the collector electrode, where it is absorbed.

To decrease the numerical burden, these computations have been carried out in a reduced domain in which the distance $H-3 a / 4$ from the origin to the collector electrode is divided by a factor of 3 , which amounts to reducing $\xi_{0}$ in (2.12) by a factor of $3^{1 / 2}$. The voltage applied between the electrodes is adjusted in order to keep the harmonic field around the injection electrode unchanged. Since the harmonic 

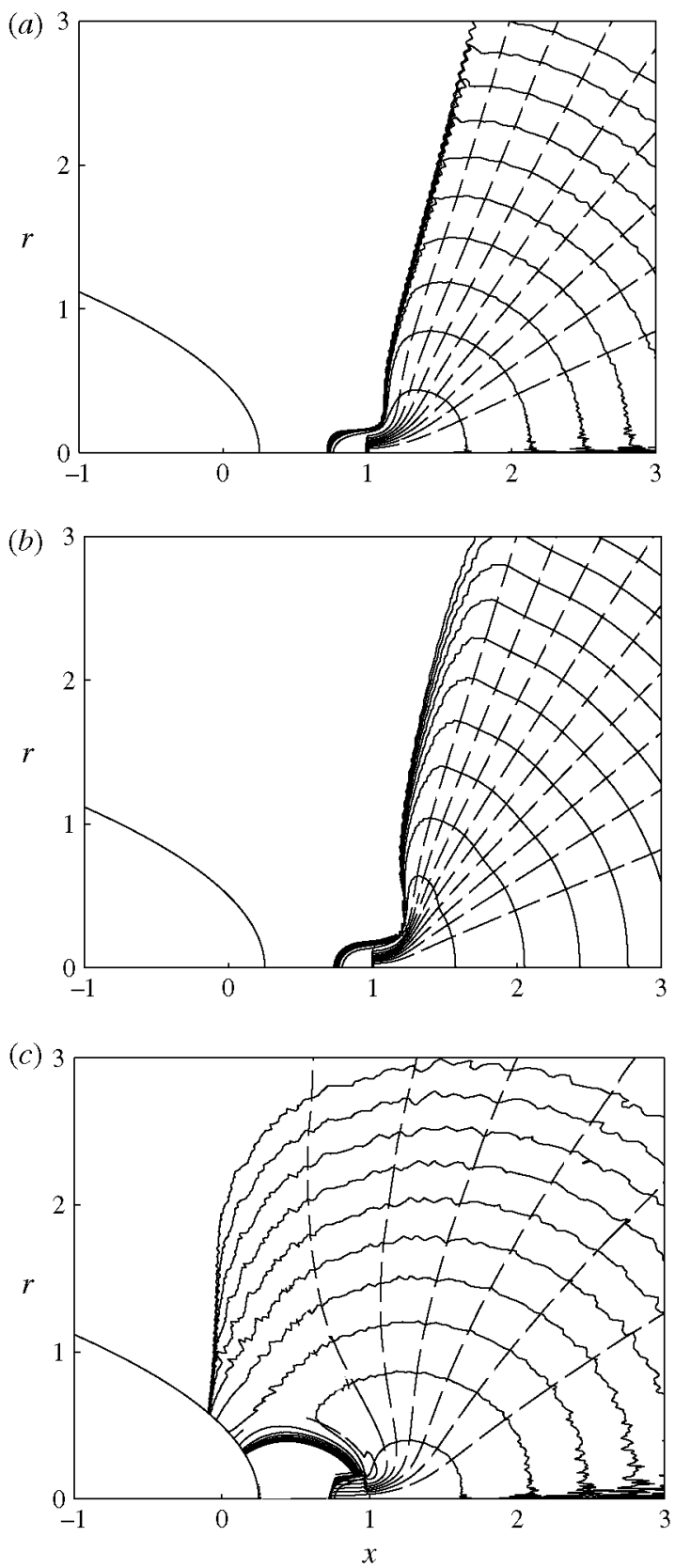

FIGURE 4. (Colour online) Contours of $n_{p}$ (solid, black) and streamlines of the flow of droplets (dashed, shown in red online) in the vicinity of the injection electrode for the modified boundary condition discussed in the text, with $\Lambda=0.1148, \Pi=1.5440$, $\boldsymbol{v}_{I}=0.7093$ and $H=20.5$. (a) Results of the particle method. (b) Results of the Eulerian method. (c) Results of the particle method with the original electrical boundary condition in (2.10) at the injection electrode, showing fly back. 
potential between two confocal paraboloidal electrodes is $\varphi_{h}=V \ln \xi / \ln \xi_{0}$ in terms of the paraboloidal coordinates of $\$ 2$ (see, e.g., Landau \& Lifshitz 1993, chapters 1 and 2 ), the voltage must be reduced by a factor $\ln \left(\xi_{0} / 3^{1 / 2}\right) / \ln \xi_{0} \approx 0.7991$, and the scaling factors $v_{0}$ and $n_{0}$ of $\S 2$ change accordingly. The modified values of the dimensionless parameters are

$$
\Lambda=0.1148, \quad \Pi=1.5440, \quad \boldsymbol{v}_{I}=0.7093, \quad H=20.5 .
$$

The number density of droplets, averaged over $10^{5}$ time steps after the solution reaches a statistically stationary state, is shown in figure $4(a)$ and compared with the results of the Eulerian model, in figure $4(b)$, for the same values of the parameters. Figure $4(c)$ illustrates the fly back that occurs when the original boundary condition for the electric potential at the injection electrode in (2.10) is used with the particle method.

\section{Flow induced in the gas}

The flow induced in the gas by the drag of the droplets, and the effect of this flow on the evolution of the spray, are discussed in this section. A monodisperse spray is still assumed ( $\phi_{r}=0$ in (2.5), so that $n_{s}=0$ ), but with $F>0$ in (2.6).

The motion of the gas around the injection electrode is very weak and has little effect on the spray when the dimensionless droplet flux is very small, $\Lambda \ll 1$. Then the number density of droplets can be conveniently rewritten as $n_{p}=\Lambda \widetilde{n}_{p}$ with $\widetilde{n}_{p}=O(1)$. This rescaling moves the parameter $\Lambda$ from the right-hand side of equations (2.4) to the right-hand side of the Poisson equation in (2.7), and changes $F$ to $\Lambda F \ll 1$ in (2.6). Therefore, the electric field induced by the charge of the droplets is very small and can be neglected compared with the electric field due to the applied voltage, and the flow induced in the gas is very weak and $\boldsymbol{v}_{g}$ can be neglected compared with $\boldsymbol{v}_{p}$ in the momentum equation for the droplets. The solution for small $\Lambda$ in a gas at rest described at the beginning of $\S 3$ is still valid in first approximation. The small velocity of the gas can be estimated as follows. The electric force acting on the streak of charged droplets that develops downstream of the injection electrode, where the effect of the inertia of the droplets becomes negligible, is transferred to the gas and must be balanced by a viscous shear force. In orders of magnitude this balance reads $\operatorname{Re}^{-1}\left(v_{g_{x}} / \delta_{p}\right) \delta_{p} \sim \Lambda F E_{x} / v_{p_{x}}$ with $E_{x} \sim 1 / x$ and $v_{p_{x}} \sim E_{x} / \Pi$, where the estimates of $\S 3$ have been used. This gives $v_{g_{x}} \sim \Lambda F \Pi R e$ independent of $x$ in the plume that the electric force induces in the gas. The ratio $v_{g_{x}} / v_{p_{x}}$ increases with $x$. It ceases to be small, and the flow of the gas ceases to be a small perturbation to the spray, when $x \sim 1 /\left(\Lambda F \Pi^{2} R e\right)$. The small $\Lambda$ structure described in this paragraph is valid only when this distance is large compared with the interelectrode distance $H$, i.e. for $\Lambda \ll 1 /\left(F \Pi^{2} R e H\right)$, which does not include the reference case.

The distribution of $n_{p}$ computed from the numerical solution of (2.4) and (2.6)-(2.13) for $\Lambda=0.001$ and the reference values of other parameters (not displayed) is close to that of figure $2(a)$ in the vicinity of the injection electrode, although differences appear farther downstream, in agreement with the estimates above. The numerical solution of (2.4) and (2.6)-(2.13) for the reference case $(\Lambda=0.0819)$ is shown in figure 5 . As can be seen, the initial evolution of the spray, in the hatched region where the inertia of the droplets is important, is not unlike that in figure $2(b)$. Now, however, the density of charged droplets in this region is much higher than in figure $2(b)$ because the value of $\Lambda$ is more than 10 times larger. The electric field induced by this charge overcomes the applied field and makes the axial field negative in the region enclosed by the dotted contour, which extends along the shroud of 


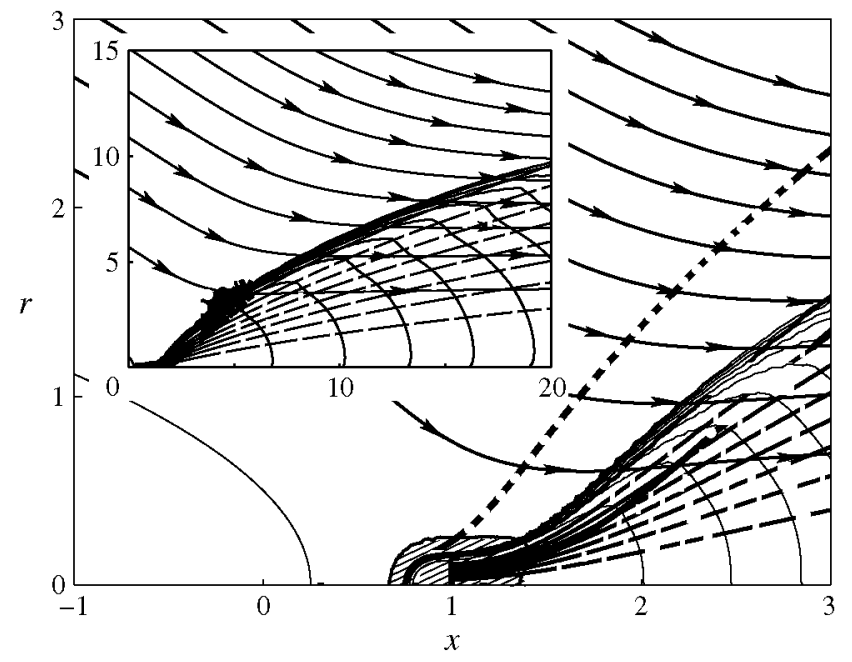

FIGURE 5. (Colour online) Contours of $n_{p}$ (solid, black), streamlines of the flow of the droplets (dashed, shown in red online) and streamlines of the flow of the gas (solid with arrows, shown in blue online), in the vicinity of the injection electrode for the reference values of the parameters. Shown are 10 equispaced contours of $n_{p}^{-1}$ between 2 and 20 . Hatched: region where $v_{p_{x}}-v_{g x}-E_{x} / \Pi$ is above $20 \%$ of its maximum. Dotted (shown in green online): boundary of the region where $E_{x}<0$.

the spray into the region where the inertia of the droplets is negligible. This would cause fly back in the absence of gas motion, but the flow of the gas carries the droplets across the region of negative axial field and beyond. This aiding flow is due to the depression associated to the entrainment of the plume that develops further downstream, where the axial electric field points again toward the collector electrode.

The balance of the viscous force and the electric force transferred to the gas by the streak of charged droplets around the axis of this plume reads now $\operatorname{Re}^{-1}\left(v_{g_{x}} / \delta_{p}\right) \delta_{p} \sim \Lambda F E_{x} / v_{g_{x}}$, which differs from the balance used earlier in this section in that $v_{p_{x}}$ has been replaced by $v_{g_{x}}$ in the estimate of the electric force. This is because the velocity of the droplets is now of the order of the velocity of the gas, so that the electric charge per unit length of the streak is of order $n_{p} \delta_{p}^{2} \sim \Lambda / v_{p_{x}} \sim \Lambda / v_{g_{x}}$. With $E_{x} \sim 1 / x$, the modified balance gives $v_{g_{x}} \sim(\Lambda F R e / x)^{1 / 2}$. The velocity of the droplets relative to the gas is $v_{p_{x}}-v_{g_{x}} \sim E_{x} / \Pi$, from (2.4), so that $\left(v_{p_{x}}-v_{g_{x}}\right) / v_{g_{x}} \sim 1 /\left(\Lambda F \Pi^{2} \operatorname{Re} x\right)^{1 / 2} \ll 1$. In the absence of mutual repulsion, the droplets would be convected essentially by the divergence-free velocity of the gas, which does not change the number density and makes the thickness of the streak proportional to the inverse of the square root of $v_{g}$. However, the charge of the droplets induces a radial field of order $n_{p} \delta_{p}$ in the streak (cf. $\S 3$ ), and therefore an additional radial velocity of order $v_{r}=n_{p} \delta_{p} / \Pi$. The kinematic condition $\delta_{p} / v_{r} \sim x / v_{g_{x}}$, already used in $\S 3$, implies that, under the action of this velocity, the thickness of the streak of droplets becomes $\delta_{p} \sim x /(F \Pi R e)^{1 / 2}$, which is in qualitative agreement with the nearly linear growth of $\delta_{p}$ with streamwise distance apparent in figure 5 away from the electrodes. The kinematic condition and the continuity equation for the gas in (2.6) also imply that the radial velocity of the gas opposes the velocity induced by the radial electric field and tends to confine the streak of droplets. The thickness of 
the gas plume is $\delta_{g} \sim\left(x^{3} / \Lambda F R e^{3}\right)^{1 / 4}$, from the balance of inertia and viscous force, $v_{g x}^{2} / x \sim v_{g_{x}} /\left(\operatorname{Re} \delta_{g}^{2}\right)$ in the momentum equation in (2.6). In the reference case, the ratio $\delta_{p} / \delta_{g} \sim\left(\Lambda \operatorname{Re} x / F \Pi^{2}\right)^{1 / 4}$ is of order unity for $x=O(H)$. The effect of the inertia of the droplets remains negligible in the plume, even though the inertia of the gas plays an important role. This is because, when $\boldsymbol{v}_{g} \sim \boldsymbol{v}_{p}$, the ratio $O\left(\boldsymbol{v}_{p} \cdot \nabla \boldsymbol{v}_{p}\right) / O\left[\Pi\left(\boldsymbol{v}_{p}-\boldsymbol{v}_{g}\right)\right]$ of the inertia to drag terms in the momentum equation for the droplets in (2.4) differs from the ratio $O\left(\boldsymbol{v}_{g} \cdot \nabla \boldsymbol{v}_{g}\right) / O\left[n_{p} F \Pi\left(\boldsymbol{v}_{p}-\boldsymbol{v}_{g}\right)\right]=O(1)$ of the analogous terms in the momentum equation for the gas in (2.6) by a factor $n_{p} F \ll 1$.

Additional numerical computations show that the size of the region of negative axial field increases when $\Lambda$ is increased keeping other parameters constant. This region extends to the apparent tip of the injection electrode containing the conical meniscus for $\Lambda \approx 0.11$, which would prevent the formation of a cone-jet and the emission of droplets. However, the numerical results do not show fly back even in these conditions when the motion of the gas is taken into account.

\subsection{Effect of the droplet-to-gas mass ratio}

When $F$ is large, the mass of droplets per unit volume is large compared with the mass of gas per unit volume in the region where $F n_{p}$ is large. The droplets provide most of the inertia of the two-fluid system in this region, and are therefore expected to account for most of the electric force. Momentum exchange with the droplets can easily accelerate the gas. For a monodisperse spray, the term $F \Pi n_{p}\left(\boldsymbol{v}_{p}-\boldsymbol{v}_{g}\right)$ in the gas momentum equation is large whenever $\left|\boldsymbol{v}_{p}-\boldsymbol{v}_{g}\right|$ is not small, causing the velocity of the gas to rapidly approach the velocity of the droplets. The droplets, on the other hand, are only weakly affected by the gas in this region; the drag term $-\Pi\left(\boldsymbol{v}_{p}-\boldsymbol{v}_{g}\right)$ in the droplets momentum equation rapidly becomes small, and the droplets evolve as they would do in a vacuum.

As the spray evolves, the mass of gas set in motion by pressure and viscosity forces increases with streamwise distance, and the number density of droplets in the spray decreases, until the contribution of the gas to the inertia of the two-fluid system becomes significant and the gas begins to play a role in the evolution of the spray. Even further downstream, the inertia of the gas dominates and the plume structure described above should be recovered.

Numerical computations have been carried out for various values of $F$ with other parameters set to the reference values in table 1. Figure 6 shows the evolution of different variables along the symmetry axis near the injection electrode. The velocity of the droplets (solid curves in figure $6 a$ ) decreases with streamwise distance beyond the injection region for small and moderate values of $F$, but increases in a leading region of the spray and reaches a maximum at $x \approx 2$ before decreasing slowly when $F=40$. The velocity in this leading region approaches that in vacuum (increasing dotted curve in the upper part of figure $6(a)$, for $\Pi=0$ in (2.4)), which is in agreement with the discussion two paragraphs above. The velocity of the gas (dashed curves in figure $6 a$ ) increases with $F$ and approaches the velocity of the droplets in the leading region, where the inertia of the droplets dominates. The length of this region increases with $F$.

The density of droplets, in figure $6(b)$, has a maximum in the injection region, whose value decreases with increasing $F$. A cone-jet cannot exist for the smallest value of $F$ in this figure, because the electric field induced by the charge of the droplets in the injection region overcomes the applied field and makes the axial electric field at the injection electrode negative. Figure 7 shows the spray plume in 

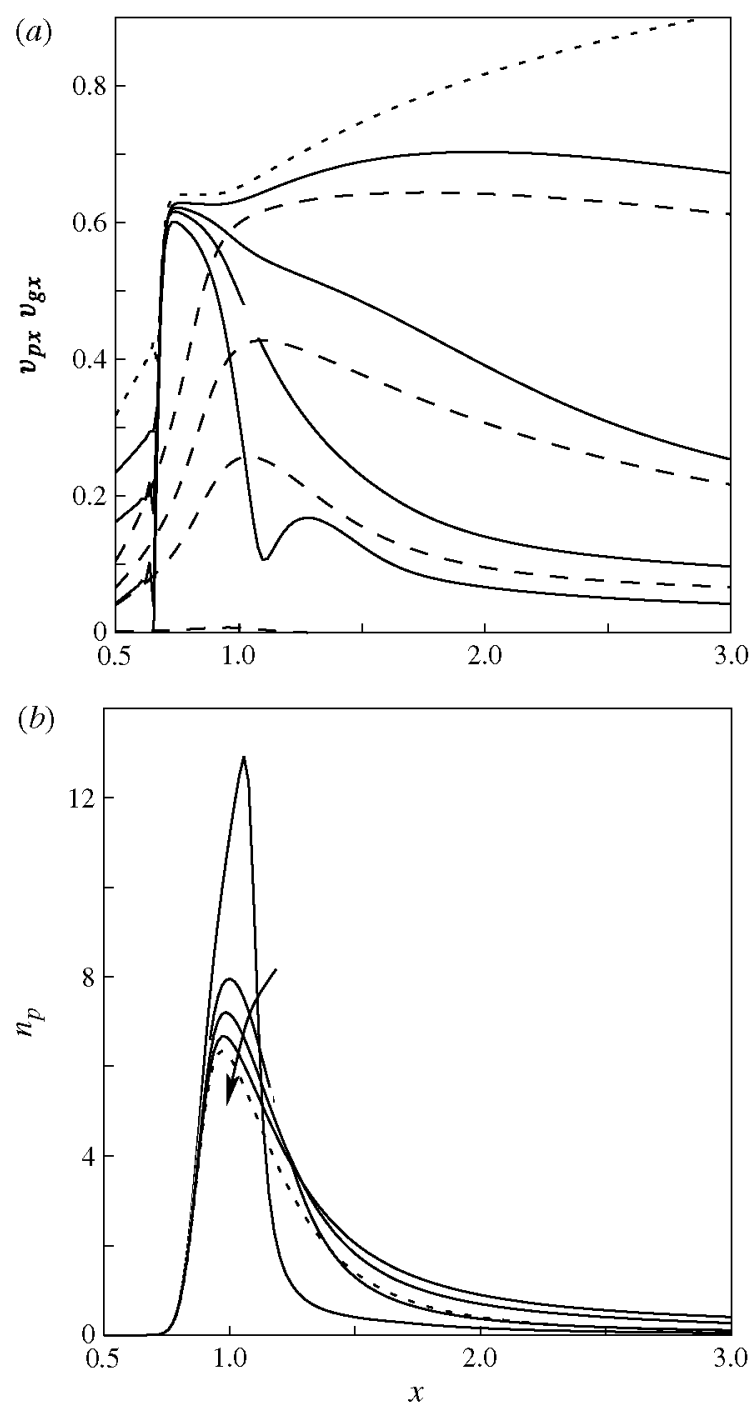

FIGURE 6. (Colour online) Droplet ( $v_{p_{x}}$, solid) and gas $\left(v_{g_{x}}\right.$, dashed) velocity, in $(a)$, and number density $\left(n_{p}\right)$, in $(b)$, along the symmetry axis for $F=0.1,4,10$ and 40 , increasing from bottom to top in $(a)$ and as indicated by the arrow in $(b)$. Other parameters have their reference values. The dotted curves give the velocity and density of the droplets in the absence of gas resistance, $\Pi=0$.

the interelectrode space. As can be seen, the thickness of the plume decreases when $F$ increases, in agreement with the estimates of $\delta_{p}$ and $\delta_{g}$ above. For $F=20$ and 40 , the confining effect of the gas makes the spray narrower than in vacuum (figure $7 d$ ).

\section{Sprays with satellite droplets}

The effect of satellite droplets has been investigated numerically for a satelliteto-primary droplet radius ratio of $1 / 3$, for which $m_{r}=(1 / 3)^{3}, q_{r}=(1 / 3)^{3 / 2}$ and $c_{r}=1 / 3$, as was mentioned at the end of $\S 2$. The numbers of primary and satellite 

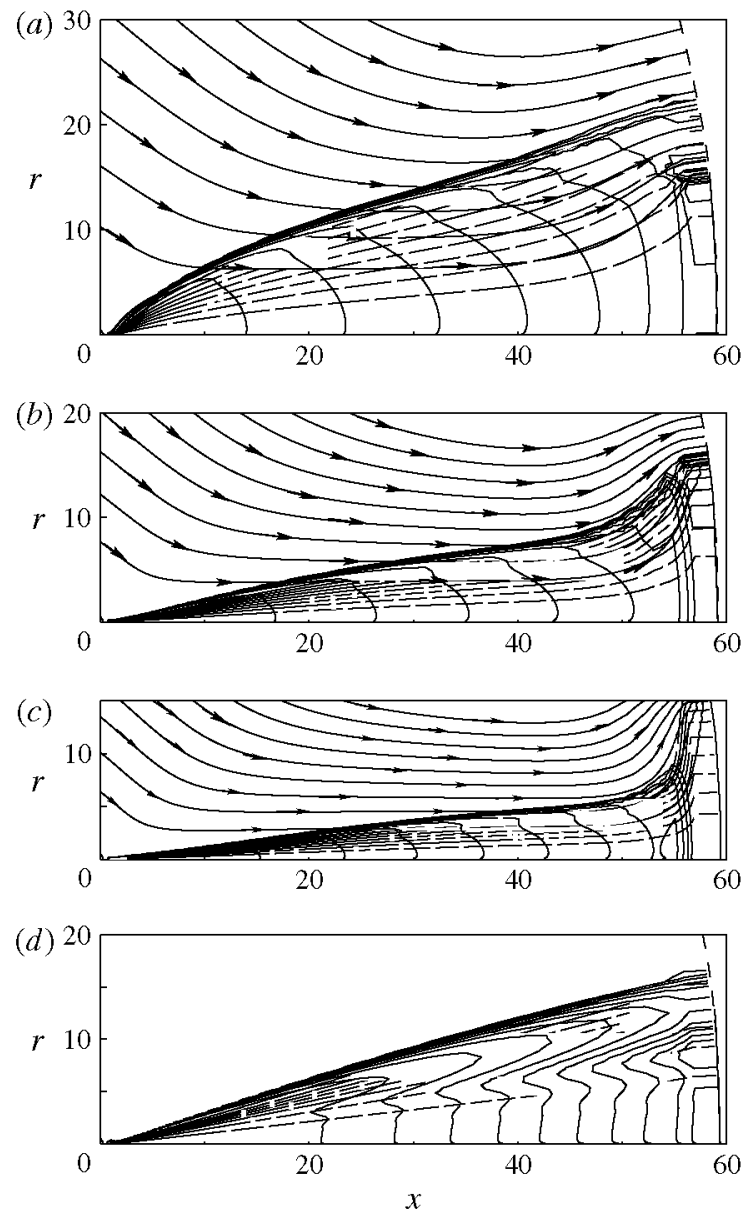

FIGURE 7. (Colour online) Contours of $n_{p}$ (solid, black), streamlines of the flow of the droplets (dashed, shown in red online), and streamlines of the flow of the gas (solid with arrows, shown in blue online), for (a) $F=5.583$, (b) $F=20$, (c) $F=40$ and (d) in the absence of gas. Other parameters have their reference values. The thick curves at the righthand sides show the collector electrode.

droplets injected per unit time are taken to be equal to each other, $\phi_{r}=1$. All other parameters are set to their reference values except for $\Lambda$, which is changed in different computations.

In the absence of gas motion $(F=0)$, the evolution of the spray with increasing $\Lambda$ is similar to that discussed in $\S 3$ for a monodisperse spray. The stopping distance is smaller for satellite droplets than for primary droplets. As noted by Tang \& Gomez (1994), satellite droplets begin to follow the electric field when the effect of the inertia of the primary droplets is still important and keeps these droplets moving nearly parallel to the symmetry axis. This difference tends to confine the primary droplets to the core of the spray, while satellite droplets are displaced to its shroud already in the initial evolution of the spray. This segregation is illustrated in figure $8(a)$ for $\Lambda=0.008$. The segregation persists further downstream, where the inertia of the primary droplets also becomes negligible, because the radial electric field induced by 

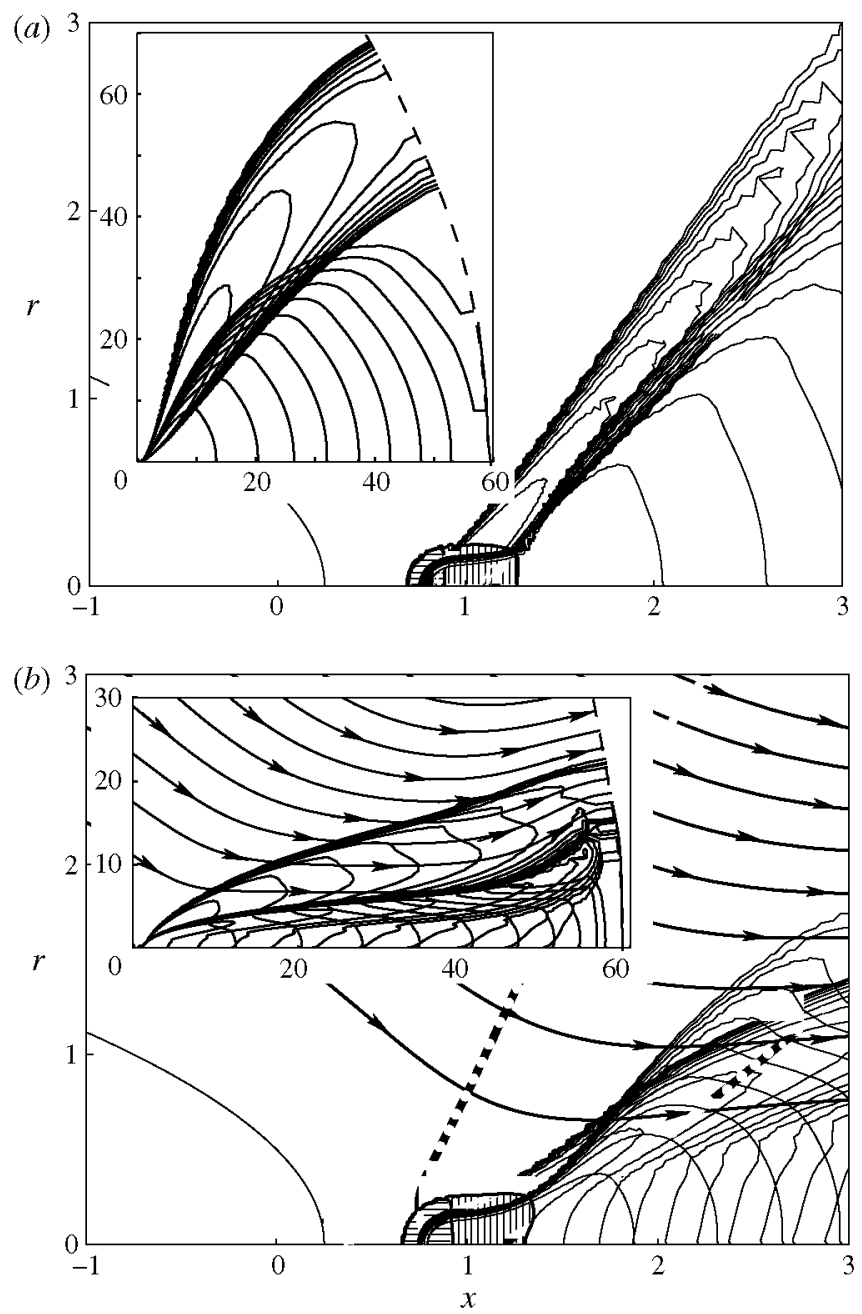

FIGURE 8. (Colour online) Contours of $n_{p}$ (black) and $n_{s}$ (shown in red online). In (a), $\Lambda=0.008, F=0$, and other parameters have their reference values in table 1 . In $(b)$, all of the parameters have their reference values, the solid curves with arrows (shown in blue online) are streamlines of the gas, and the dotted contour (shown in green online) is the boundary of the region where $E_{x}<0$. Shown are 10 equispaced contours of $n_{p}^{-1}$ between 10 and 100 in $(a)$ and between 1.818 and 18.18 in $(b)$, and 10 equispaced contours of $n_{s}^{-1}$ between 2 and 20 in (a) and between 1.429 and 14.29 in (b). Hatched: regions where $v_{p_{x}}-v_{g_{x}}-E_{x} / \Pi$ (vertical hatching) and $v_{s x}-v_{g_{x}}-\left(q_{r} / c_{r}\right) E_{x} / \Pi$ (horizontal hatching) are above $20 \%$ of their respective maxima.

the charge of the droplets is larger at the shroud than in the core of the spray (Tang \& Gomez 1994; Hartman et al. 1999). The value of $\Lambda$ in figure $8(a)$ is close to the maximum that can be attained without fly back, which, as for a monodisperse spray in $\S 3$, is much smaller than the reference value taken from Gañán-Calvo et al. (1994) experiments. Also as in $\S 3$, the axial field first takes negative values (toward the injection electrode) where the effect of the inertia of the primary droplets declines. Since the inertia of the satellite droplets is also negligible in this region, both types of droplets begin to fly back nearly at the same time when $\Lambda$ is increased. 
Important changes occur when the motion of the gas is taken into account using the reference values of $F$ and $R e$. As for a monodisperse spray, the flow of the gas induced by the entrainment of the electrohydrodynamic plume allows the droplets to overcome the negative axial electric field that appears in the injection region and the shroud of the spray when $\Lambda$ increases. No fly back occurs. The cone-jet mode fails because the electric field becomes negative in the region containing the meniscus when $\Lambda$ becomes larger than $\sim 0.09$.

The motion of the gas also affects droplet segregation. Figure $8(b)$, for the reference case, shows that satellite droplets initially decelerate and tend to move to the shroud of the spray, but this trend is reversed further downstream and satellite droplets end up concentrating in an intermediate radial layer which is surrounded by primary droplets on both sides. This result can be understood noticing that the momentum equations for primary and satellite droplets reduce to $\boldsymbol{v}_{p}-\boldsymbol{v}_{g}=\boldsymbol{E} / \Pi$ and $\boldsymbol{v}_{s}-\boldsymbol{v}_{g}=\left(q_{r} / c_{r}\right) \boldsymbol{E} / \Pi$ when the inertia of both types of droplets is negligible. The drift of the droplets relative to the gas is proportional to the electric field, but the drift velocity is smaller for satellite droplets than for primary droplets because $q_{r} / c_{r}<1$. Satellite droplets nearly follow the gas, whose radial velocity (not displayed) is positive around the symmetry axis, due to the deceleration of the gas and the droplets, and negative in the outermost part of the spray, due to the entrainment it induces. This explains the concentration of satellite droplets in an intermediate layer, which is partially depleted of primary droplets due to Coulomb repulsion forces.

Consider now the limit $F \rightarrow \infty$. The terms $F \Pi n_{p}\left(\boldsymbol{v}_{p}-\boldsymbol{v}_{g}\right)$ and $F \Pi C_{r} n_{s}\left(\boldsymbol{v}_{s}-\boldsymbol{v}_{g}\right)$ in the momentum equation for the gas (2.6) are large in a certain region around the injection electrode when $F \gg 1$. The gas in this region rapidly approaches the velocity $\boldsymbol{v}_{g}=\left(n_{p} \boldsymbol{v}_{p}+c_{r} n_{s} \boldsymbol{v}_{s}\right) /\left(n_{p}+c_{r} n_{s}\right)$ that makes the sum of these two terms equal to zero. However, none of the drag terms $-\Pi\left(\boldsymbol{v}_{p}-\boldsymbol{v}_{g}\right)=-\Pi c_{r} n_{s}\left(\boldsymbol{v}_{p}-\boldsymbol{v}_{s}\right) /\left(n_{p}+c_{r} n_{s}\right)$ and $-\left(c_{r} / m_{r}\right) \Pi\left(\boldsymbol{v}_{s}-\boldsymbol{v}_{g}\right)=-\left(c_{r} / m_{r}\right) \Pi n_{p}\left(\boldsymbol{v}_{s}-\boldsymbol{v}_{p}\right) /\left(n_{p}+c_{r} n_{s}\right)$, in the momentum equations (2.4) and (2.5) for primary and satellite droplets, is then small. In contrast to the case of a monodisperse spray, this means that the gas plays now a role in the evolution of the droplets, as it mediates an interaction between droplets of different sizes additional to the electrostatic interaction. The velocities of the primary and satellite droplets and of the gas along the symmetry axis are shown in figure 9 for various values of $F$. In the region displayed, the velocity of the primary droplets (solid curves) becomes of the order of the velocity that these droplets would have in vacuum (dotted curve, for $\Pi=0$ ) when $F$ increases. The velocity of the satellite droplets (chain curves) is much smaller than in vacuum, where energy conservation makes the velocity of these droplets larger than the velocity of the primary droplets by a factor of order $\left(q_{r} / m_{r}\right)^{1 / 2}$. The difference shows that the effect of the inertia of the satellite droplets is small in this region. The momentum equation for these droplets reduces to $c_{r} \Pi\left(\boldsymbol{v}_{s}-\boldsymbol{v}_{g}\right) \approx q_{r} \boldsymbol{E}$, whence, upon using the expression of $\left(\boldsymbol{v}_{p}-\boldsymbol{v}_{g}\right)$ above and carrying the result to the right-hand side of the momentum equation for the primary droplets, the force on these droplets reduces to $\left(1+q_{r} n_{s} / n_{p}\right) \boldsymbol{E}$. The combined effect of the gas and the satellite droplets leads thus to an apparent increase of the charge of the primary droplets in the region where the mass of droplets per unit volume is large compared to the mass of gas per unit volume and the approximations at the beginning of this paragraph apply. 


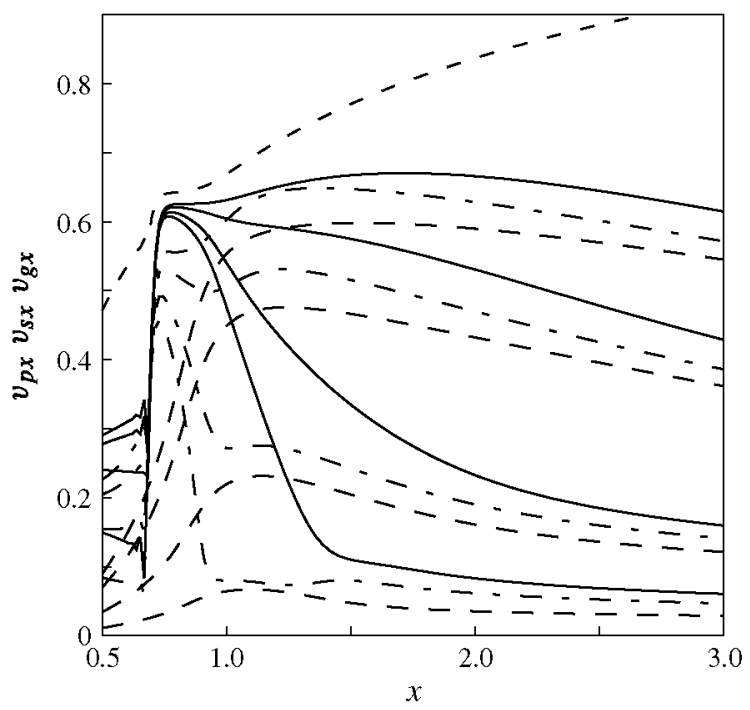

FIGURE 9. (Colour online) Velocities of the primary droplet ( $v_{p_{x}}$, solid), the satellite droplets $\left(v_{s_{x}}\right.$, chain) and the gas ( $v_{g_{x}}$, dashed) along the symmetry axis for $\Lambda=0.02$ and $F=2,10$, 40 and 80 , increasing from bottom to top. Other parameters have their reference values. The dotted curve in the upper part of the figure gives the velocity of the primary droplets in the absence of gas resistance, $\Pi=0$.

\section{Conclusions}

The dynamics of an aerosol of electrically charged droplets, and the flow induced in the surrounding gas by the drag of the droplets, are computed using a multifluid Eulerian model that takes into account the inertia of the droplets and the gas, the electric forces acting on the droplets, and the aerodynamic forces due to the motion of the droplets relative to the gas. The aerosol is generated by an electrospray source that emits primary and satellite droplets, and these droplets travel from the source to a collector electrode in font of the source. The voltage applied between the source and the collector, together with the electric charge of the droplets, determine the electric field in the interelectrode space.

Results for a monodisperse spray in a quiescent gas show that the axial electric field induced by the charged droplets is small compared with the field due to the applied voltage when the flux of droplets is very small, in agreement with the results of Tang \& Gomez (1994) and others. The two contributions to the axial field become of the same order, and oppose each other in the vicinity of the injection electrode, when the dimensionless flux becomes of order $\Lambda_{c}=1 /(\Pi H)$. Then the spray rapidly broadens when the effect of the inertia of the droplets declines, slightly downstream of the region where the droplets are generated in the model. When $\Lambda$ increases, the electric field first becomes negative (toward the injection electrode) in the region where the spray broadens, and negative velocities, leading to fly back of the droplets toward the injection electrode and to the breakdown of the Eulerian model, occur above a critical value of $\Lambda$ of the order of $\Lambda_{c}$. This critical value computed for a gas at rest is small compared with the values of $\Lambda$ reported in the experiments of Gañán-Calvo et al. (1994) and Tang \& Gomez (1994). The critical value of $\Lambda$ increases when the effect 
of the image charge in the injection electrode is omitted, as has been often done in Lagrangian simulations.

The flow induced in the gas plays an important role when the dimensionless flux is of order $1 /\left(F \Pi^{2} R e\right)$ or larger, which includes the conditions of these experiments. The drag of the droplets generates a plume of gas at some distance from the injection electrode. The entrainment of this plume convects the droplets with a velocity large compared with the axial velocity directly induced by the electric field, which allows the droplets to cross the region of negative axial field that appears around the origin of the spray when the flux of droplets increases. This suppresses fly back and allows to reach the fluxes reported in the experiments of Gañan-Calvo et al. (1994) and Tang \& Gomez (1994). A new limitation appears for even larger fluxes, when the region of negative axial field extends to the electrospray source. The radial velocity of the gas opposes the radial velocity of the droplets relative to the gas due to the radial electric field induced by the droplets. As a consequence, the radius of the spray is smaller than in a quiescent gas and increases linearly with streamwise distance. For large values of the droplet-to-gas mass ratio, measured by the parameter $F$, the resistance of the gas is negligible, and the velocity of the gas rapidly adjust to the velocity of the droplets, in a leading region of the spray whose length increases with $F$.

Size segregation, whereby satellite droplets migrate to the shroud of the spray, is reproduced by the Eulerian model and is also affected by the motion of the gas. Owing to their smaller charge to friction coefficient ratio, the velocity of the satellite droplets relative to the gas is smaller than the velocity of the primary droplets relative to the gas when the inertia of both types of droplets becomes negligible. Since the radial velocity of the gas is negative (toward the axis) in the shroud of the spray, the combination of this flow and the differential mobility opposes the electrostatic/inertial mechanism of segregation and may cause satellite droplets to concentrate in an intermediate radial region of the spray. The gas mediates an interaction between primary and satellite droplets in the leading region of the spray there the effect of the gas inertia is small when $F \gg 1$. If the inertia of the satellite droplets is also small in this region, the interaction amounts to an apparent increase of the charge of the primary droplets.

\section{Acknowledgements}

This work was supported through projects DPI2010-20450-C03-01 and CSD201000010 (Ministerio de Economía y Competitividad), and S2009/ENE-1597 (Comunidad de Madrid).

\section{REFERENCES}

Barrero, A. \& Loscertales, I. G. 2007 Micro- and nanoparticles via capillary flows. Annu. Rev. Fluid Mech. 19, 89-106.

Birdsall, C. K. \& Langdon, A. B. 1981 Plasma Physics Via Computer Simulation. Taylor and Francis.

Clift, R., Grace, J. R. \& Weber, M. E. 1978 Bubbles, Drops and Particles. Academic.

Cloupeau, M. \& Prunet-Foch, B. 1989 Electrostatic spraying of liquids in cone-jet mode. J. Electrostat. 22, 135-159.

DENG, W. \& GOMEZ, A. 2007 Influence of space charge on the scale-up of multiplexed electrosprays. J. Aerosol Sci. 38, 1062-1078.

Fenn, J. B., Mann, M., Meng, C. K., Wong, S. K. \& Whitehouse, C. M. 1989 Electrospray ionization for mass spectrometry of large biomolecules. Science 246, 64-71. 
FERNÁNDEZ DE LA MORA, J. 1992 The effect of charge emission from electrified liquid cones. J. Fluid Mech. 243, 561-574.

Fernández de la Mora, J. 2007 The fluid dynamics of Taylor cones. Annu. Rev. Fluid Mech. 39, $217-243$.

FILIPPOV, A. V. 1991 Electrostatic deposition of inertially moving charged aerosol particles onto the earthed disk. J. Electrostat. 26, 81-98.

Filippov, A. V. 1992 Electrostatic deposition of a moving charged aerosol cloud onto a conducting sphere. J. Aerosol Sci. 23, 203-215.

Gamero-Castaño, M. 2008 The structure of electrospray beams in vacuum. J. Fluid Mech. 604, $339-368$.

Gañán-Calvo, A. M., Lasheras, J. C., Dávila, J. \& Barrero, A. 1994 Electrostatic spray emitted from an electrified conical meniscus. J. Aerosol Sci. 25, 1121-1142.

GRACE, J. M. \& Dunn, P. F. 1996 Droplet motion in an electrohydrodynamic fine spray. Exp. Fluids 20, 153-164.

GRIFOLL, J. \& ROSELL-Llompart, J. 2012 Efficient Lagrangian simulation of electrospray droplets dynamics. J. Aerosol Sci. 47, 78-93.

Hartman, R. P. A., Borra, J.-P., Brunner, D. J., Marijnissen, J. C. M. \& Scarlett, B. 1999 The evolution of electrohydrodynamic sprays produced in the cone-jet mode, a physical model. J. Electrostat. 47, 143-170.

Higuera, F. J. 2012 Eulerian model of a dilute spray of charged droplets. J. Aerosol Sci. 48, 34-45.

Hockney, R. W. \& Eastwood, J. W. 1988 Computer Simulation Using Particles. Taylor and Francis.

JACKSON, R. 1997 Locally averaged equations of motion for a mixture of identical spherical particles and a Newtonian fluid. Chem. Engng Sci. 52, 2457-2469.

JACKSON, R. 2000 The Dynamics of Fluidized Particles. Cambidge University Press.

Landau, L. D. \& Lifshitz, E. M. 1981 Physical Kinetics. Pergamon.

LANDAU, L. D. \& LifShitZ, E. M. 1993 Electrodynamics of Continuous Media. Pergamon.

LEE, Y. H., Kimm, C. A., JANG, W. H., ChOI, H. J. \& JHON, M. S. 2001 Synthesis and electrorheological characteristics of microencapsulated polyaniline particles with melamine-formaldehyde resins. Polymer 42, 8277-8283.

OH, H., Kıм, K. \& KIM, S. 2008 Characterization of deposition patterns produced by twin-nozzle electrospray. J. Aerosol Sci. 39, 801-813.

TANG, K. \& GOMEZ, A. 1994 On the structure of an electrostatic spray of monodisperse droplets. Phys. Fluids 6, 2317-2332.

TAYLOR, G. I. 1964 Disintegration of water drops in an electric field. Proc. R. Soc. Lond. A 280, 383-397.

Wilhelm, O., MÄDler, L. \& PRAtsinis, S. E. 2003 Electrospray evaporation and deposition. J. Aerosol Sci. 34, 815-836.

YANG, W., LOJEWSKi, B., WEI, Y. \& DENG, W. 2012 Interactions and deposition patterns of multiplexed electrosprays. J. Aerosol Sci. 46, 20-33.

Zeleny, J. 1917 Instabilities of electrified surfaces. Phys. Rev. 10, 1-6.

Zhang, D. Z. \& Prosperetti, A. 1997 Momentum and energy equations for disperse two-phase flows and their closure for dilute suspensions. Intl J. Multiphase Flow 23, 425-453. 